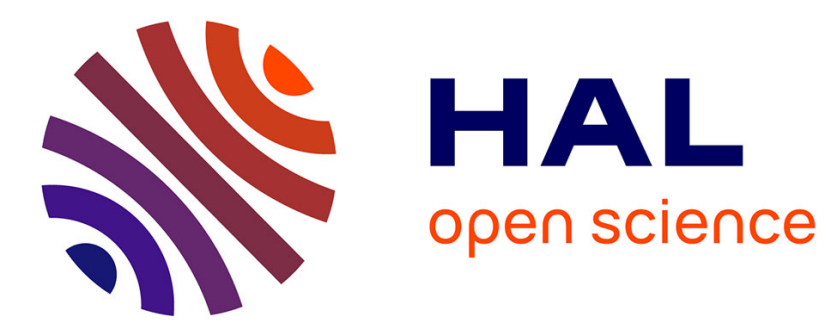

\title{
Experimental nanocalorimetry of protonated and deprotonated water clusters
}

Julien Boulon, Isabelle Braud, Sébastien Zamith, Pierre Labastie, Jean-Marc

L'Hermite

\section{- To cite this version:}

Julien Boulon, Isabelle Braud, Sébastien Zamith, Pierre Labastie, Jean-Marc L'Hermite. Experimental nanocalorimetry of protonated and deprotonated water clusters. Journal of Chemical Physics, 2014, 140, pp.164305. 10.1063/1.4871882 . hal-00983145

\section{HAL Id: hal-00983145 \\ https://hal.science/hal-00983145}

Submitted on 24 Apr 2014

HAL is a multi-disciplinary open access archive for the deposit and dissemination of scientific research documents, whether they are published or not. The documents may come from teaching and research institutions in France or abroad, or from public or private research centers.
L'archive ouverte pluridisciplinaire HAL, est destinée au dépôt et à la diffusion de documents scientifiques de niveau recherche, publiés ou non, émanant des établissements d'enseignement et de recherche français ou étrangers, des laboratoires publics ou privés. 


\title{
Experimental nanocalorimetry of protonated and
}

\section{deprotonated water clusters.}

\author{
Julien Boulon $^{1,2}$, Isabelle Braud ${ }^{1,2}$, Sébastien Zamith ${ }^{1,2}$, Pierre Labastie ${ }^{1,2}$, and Jean-Marc \\ L'Hermite $^{1,2}$ \\ ${ }^{1}$ Université de Toulouse ; UPS ; Laboratoire Collisions Agrégats Réactivité, IRSAMC ; F-31062 \\ Toulouse, France \\ ${ }^{2}$ CNRS ; UMR 5589 ; F-31062 Toulouse, France
}

\begin{abstract}
An experimental nanocalorimetric study of mass selected protonated $\left(\mathrm{H}_{2} \mathrm{O}\right)_{n} \mathrm{H}^{+}$and deprotonated $\left(\mathrm{H}_{2} \mathrm{O}\right)_{\mathrm{n}-1} \mathrm{OH}^{-}$water clusters is reported in the size range $n=20-118$. Water cluster's heat capacity exhibit a change of slope at size dependent temperatures varying from 90 to $140 \mathrm{~K}$, which is ascribed to phase or structural transition. For both anionic and cationic species, these transition temperatures strongly vary at small sizes, with higher amplitude for protonated than for deprotonated clusters, and change more smoothly above roughly $n \approx 35$. There is a correlation between bonding energies and transition temperatures, which is split in two components for protonated clusters while only one component is observed for deprotonated clusters. These features are tentatively interpreted in terms of structural properties of water clusters.
\end{abstract}

\section{Introduction}


The complex behavior of bulk water is still far to be fully understood, and the properties of water clusters at the molecular scale remain even more widely unknown. It is of fundamental interest to investigate the properties of water in the molecular size range: On the one hand, it is a unique benchmark to put to the test the descriptions of water molecules in interaction; on the other hand, knowing the properties of small water clusters is intrinsically useful since they are expected to play a crucial role in ubiquitous physical phenomena, from droplets nucleation $[1,2,3]$ to liquid/solid transition in the bulk phase [4].

A great deal still has to be done to know comprehensively the properties of water clusters of more than a few molecules. The complex intermolecular interactions at play (in particular due to hydrogen bonding [5]) makes the computations particularly cumbersome and the predictions highly model dependent [6], which restricts reliable calculations to very small clusters.

Experimentally, the physical properties of water clusters were mainly investigated through sizedependent abundance study in mass spectra of both positively charged $[7,8]$ and negatively charged [9] species, through infrared (IR) spectroscopy [10] and using photoelectron spectroscopy [11]. Electron diffraction experiments performed in the 1980's suggested that water clusters become crystalline in the size range 200-1000 molecules [12]. This result was confirmed by vibrational IR spectroscopy experiments on pure water and Na-doped clusters $[10,13]$, which suggest an onset of crystallization at about 275 water molecules [13]. Computer simulations support the assumption of amorphous-tocrystalline transition occurring above 200 molecules [14]. On the other hand, 21-molecule water clusters and sizes slightly above are likely to form more or less deformed cage structure; thus, a transition from cage to amorphous structures is expected to occur somewhere between 21 and 275 molecules.

Since the pioneer work by Haberland's group [15] and the development of related methods $[16,17,18]$, nanocalorimetric studies of a few atomic (Tin [19], gallium [20], aluminum [21]) and molecular (sodium chloride [22]) clusters have been performed by recording their caloric curves, which allows identifying phase transitions or any temperature-dependent phenomena with a latent 
heat. The first order phase transitions can be identified without ambiguity when the associated peak in the caloric curves can be recorded over a sufficiently broad temperature range [16-18]. Nanocalorimetry of water clusters is still poorly documented. In the experiments recently devoted to water clusters $[23,17,24]$, no melting transition can be clearly identified since evaporation occurs too close to the transition, which prevents from observing a whole peak that would be the undeniable signature of a first order phase transition; however, the change of slope observed in all caloric curves can be ascribed to the lower edge of a phase transition. These experiments are restricted to a limited size range and to a single charge state, either positive [17] or negative [23,24], which did not bring reliable information about the size evolution of caloric curves nor allows a comprehensive comparison between anions and cations.

The present work completes previous studies and allows a comprehensive comparison of protonated and deprotonated clusters over an extended size range. All data being recorded using the same experimental setup and all heat capacities being extracted from raw data with the same method [18] enhances the robustness of the comparisons from size to size and between anions and cations.

\section{Experimental determination of water cluster's caloric curves}

A method based on clusters-atoms/molecules collisions was developed in our group to study the phase transitions of mass selected clusters through the observation of their caloric curves [18].This method has already been successfully used to study the melting phase transition of sodium clusters [18] and more recently to measure the caloric curves of deprotonated water clusters [24].The same method is again applied here to study both protonated and deprotonated water clusters.

The experimental setup and the data processing method used to extract caloric curves from the data collected in our collision experiment were described in details in previous publications [24,18]. Protonated or deprotonated water clusters are produced in a gas aggregation source and ionized in a discharge. They can be thermalized between $25 \mathrm{~K}$ and ambient temperature in a heat bath. Then, they are mass-selected, focused in energy, and slowed down before entering a collision cell containing 
water vapor. The collisional products are mass analyzed in a second time of flight mass spectrometer; the mass spectra consist of several peaks corresponding to the intact parent clusters and to clusters that have undergone attachment and/or evaporation of water molecules. The heat capacity is extracted from these spectra using the method detailed in reference [24] and reference [18]. The basic idea is to take advantage of the correlation (due to the relation between unimolecular evaporation rate and internal energy) between the barycenter of the final mass distribution and cluster's internal energy. A differential method is used: A mass spectrum is recorded at a given cluster temperature $T_{l}$ and a given collision energy $E_{c l}$; a second mass spectrum is recorded at higher collision energy $E_{c 2}=E_{c 1}+\delta E$, and the cluster temperature $T_{2}=T_{1}-\delta T$ is set so that the barycenters are identical in both experiments. The fulfillment of this condition indicates that the internal energy decrease from $T_{1}$ to $T_{2}$ has been compensated by collision energy increase $\bar{i} \delta \mathrm{E}$ ( $\bar{i}$ is the average number of collisions). The heat capacity $C(T)$ is deduced from these two experiments as $C(T) \approx \bar{i} \delta E / \delta T$. A more rigorous treatment gives the following expression of the heat capacity [24]:

$$
C(T)=-\bar{i}\left(1+\frac{\partial^{2} \bar{n}}{\partial T \partial \bar{i}} / \frac{\partial \bar{n}}{\partial T}\right) \frac{\delta E_{t}}{\delta T}
$$

Where $\bar{n}$ is the barycenter of the mass distribution, and $\delta T$ is the temperature shift that gives the same barycenter at $\left(T_{1}, E_{c l}\right)$ and $\left(T_{2}, E_{c 2}\right) . \delta E_{t}$ is given by $\delta E_{t}=\chi \delta E c+\alpha \times(1-\chi) \delta E_{c}$, where $\delta E_{c}=E_{c 1}-$ $E_{c 2}$. Measurements of attachment cross sections on water molecules onto water clusters have shown that only a part $\chi$ of the collisions, which varies with cluster's size and collision energy, leads to attachment $(\chi$ is the ratio of the attachment cross section to the collision cross section) $[25,26]$. For the other inelastic collisions, only a fraction $\alpha$ of the collision energy is converted into cluster's internal energy. The fraction of collision energy transferred to the cluster can be estimated in the frame of the impulsive collision model [27,28], which gives the value $\alpha=1 / 2$. We have taken the experimental values of $\chi$ from Ref.[25]. 
The experimental conditions are chosen in order to minimize noise and overall uncertainties. Under typical working pressures in the collision cell (between $310^{-4}$ and $910^{-4} \mathrm{mbar}$ ), the average number of collisions $\bar{i}$ varies from 1 to 6 and the barycenter $\bar{n}$ varies within a few units (in $\mathrm{H}_{2} \mathrm{O}$ mass units) with respect to the parent cluster. The couples of clusters kinetic energies $\left(E_{k 1}, E_{k 2}\right)$ were chosen between $10 \mathrm{eV}$ and $38 \mathrm{eV}$ in the laboratory frame, with $E_{k 2}-E_{k 1}$ of the order of $6 \mathrm{eV}$. The corresponding collision energies in the center of mass $(\mathrm{CM})$ frame $E_{c 1}$ and $E_{c 2}$, which depend on the size considered, are thus kept below $1 \mathrm{eV}$.

The evolution of the barycenter is plotted in figure 1 as a function of the number of collisions for $\left(\mathrm{H}_{2} \mathrm{O}\right)_{118} \mathrm{H}^{+}$colliding with water molecules at a $\mathrm{CM}$ collision energy of $0.32 \mathrm{eV}$. This experiment was performed at different initial cluster's temperatures $T_{t h}$. Such curves are used to determine at which pressure one should work to measure caloric curves. Two main aspects are used to select the working pressure. Firstly, one should work at pressure high enough so that evaporation has already set in. Evaporation manifests itself in these curves when the barycenter does not any more increase linearly with the number of collisions. As the initial temperature of the clusters increases, the number of collisions needed to induce evaporation becomes smaller and smaller. The other point to consider in choosing the pressure is to minimize the term $\frac{\partial^{2} \bar{n}}{\partial T \partial \bar{i}}$ in equation (1). Assuming a constant derivative with respect to temperature over the range considered, this term is evaluated as $\frac{\partial^{2} \bar{n}}{\partial \bar{i} \partial T} \approx \frac{\partial \bar{n} / \partial \bar{i}\left(T_{t h 2}\right)-\partial \bar{n} / \partial \bar{i}\left(T_{t h 1}\right)}{T_{t h 2}-T_{t h 1}}$ (see figure 1 and reference [18]). In the experiment on $\left(\mathrm{H}_{2} \mathrm{O}\right)_{118} \mathrm{H}^{+}$ presented in figure $1, \frac{\partial^{2} \bar{n}}{\partial T \partial \bar{i}}$ is about $10^{-3}$ leading to a correction in $C(T)$ that varies between -1 and $-4 \%$ over the whole temperature range. All along the experiments presented in this paper, it has been carefully checked that the term $\frac{\partial^{2} \bar{n}}{\partial T \partial \bar{i}} / \frac{\partial \bar{n}}{\partial T}$ introduces a correction of at most $\pm 5 \%$ in $C(T)$; such a correction is smaller than the overall uncertainty and it significantly increases the noise [18], so we chose to neglect it here. 
The way in which heat capacity is extracted from raw data is exemplified in figure 2 in the case of $\left(\mathrm{H}_{2} \mathrm{O}\right)_{55} \mathrm{H}^{+}$. In order to check the robustness of experimental observations, several experimental runs were performed for each size. All heat capacities presented here were averaged over several experiments. Following the same procedure as in reference [24], the onset of evaporation was measured at every size in order to define the range of validity of our measurements (see for example the inset in figure 2).

\section{Results}

In water clusters, unimolecular evaporation occurring at temperatures close to melting temperatures prevents from recording the heat capacity over a sufficiently broad temperature range, so no complete peak - which would unambiguously denote a first order phase transition - can be observed [23]. However, all heat capacities of water clusters observed so far, by other groups $[23,17]$ as well as in our lab [24], exhibit a noticeable change of slope at some "transition" temperature that depends on the size and of the charge state. No information is experimentally available concerning the very nature of this transition, which may be either melting transitions, glass transitions, or structural solid-solid transition $[17,24]$. Theoretical works suggest that the transition from solid to liquid of water clusters proceeds through a succession of solid-solid structural transitions spread over a broad temperature range $[29,30]$. In this paper, we prefer to call "transition temperatures", rather than "melting temperatures", the temperatures at which the heat capacities start increasing noticeably, considering, in agreement with other studies [23,17], that this feature is likely to be the lower edge of a structural change. Identifying the transition temperature as the change of slope underestimates this temperature. Moreover, the sharpness of the transition is size-dependent and is also likely to vary with the structure of clusters. Thus, we will focus on the onset of the phase transition, which is the only robust parameter available from our experiments that allows comparing the properties of clusters from size to size and from one charge state to the other. Our analysis of experimental data deal mainly with the size evolutions of the transition temperatures thus defined and with their variations from anions to cations. 


\section{Heat capacity of protonated water clusters}

The heat capacities of protonated water clusters $\left(\mathrm{H}_{2} \mathrm{O}\right)_{\mathrm{n}=20-118} \mathrm{H}^{+}$are displayed in figure 3 . The noise level is responsible for small features such as small secondary peaks at low temperature that must be considered with caution. The absolute value of the heat capacities might be overestimated or underestimated by a factor 2 [24]. Cluster's evaporation was taken into account in data processing as it has been done previously [24], namely we do not analyze the heat capacities above a given size dependent temperature from which evaporation dominates. Only reproducible features, which were confirmed by recording each curve several times, are analyzed below.

At low temperature, the heat capacities, which are lower than bulk values [31] (as for deprotonated clusters [24]) steadily increase. Between $78 \mathrm{~K}$ and $136 \mathrm{~K}$, depending on size, the heat capacities more or less suddenly increase. The transition is clear for $n=20,21$ and above $n \approx 35$, whereas it is smoother in the size range $n \approx 22-35$. Between $n=22$ and $n=35$, the maximum value reached by the heat capacity is, on average, lower than for other sizes.

At the highest temperatures investigated here, the heat capacities of waters clusters vary, depending on their size, between about $5 \mathrm{k}_{\mathrm{B}}$ and $10 \mathrm{k}_{\mathrm{B}}$. These values are higher than in bulk ice at the same temperature (around $2.5 \mathrm{k}_{\mathrm{B}} /$ molecule [31]); they are also higher than the bulk value for glassy water (of the order of $3 \mathrm{k}_{\mathrm{B}} /$ molecule) or liquid water, whose putative heat capacity is almost the same as the one of glassy water at this temperature [32]. The fact that around $150 \mathrm{~K}$ the heat capacity is higher than the bulk liquid value might hint towards a peak in the caloric curve, indicating a melting transition. However, solid-solid structural transitions also give rise to peaks in the caloric curves of water clusters [29], and no conclusion concerning the very nature of the phase transition can actually be drawn from the possible existence of a peak in the heat capacities.

The transition temperatures, deduced as shown in figure 2 from the caloric curves, are presented in figure 4. Our transition temperatures present an evolution similar to the ones previously measured, despite slightly lower values [17]. This might be partially explained by the different way transition 
temperatures are extracted in both works: in references [17] and [23], the transition temperature is determined from the caloric curve $E(T)$, whereas in our case it is extracted from its derivative $C(T)$, which underestimates this temperature. These difference reduces the disagreement with the results of Schmidt and coworkers [17] but increases the difference between the transition temperatures of $\left(\mathrm{H}_{2} \mathrm{O}\right)_{\mathrm{n}-1}{ }^{-}$clusters and the ones of $\left(\mathrm{H}_{2} \mathrm{O}\right)_{\mathrm{n}}{ }^{-}$clusters measured by Hock and coworkers [23].

Below $n \approx 35$ roughly, they strongly vary from one size to another. The highest transition temperature ( $136 \mathrm{~K}$ ) is reached at $n=21$ and no global trend can be identified in this size range. Above $n \approx 35$, on the contrary, the size-evolution of the transition temperatures becomes more regular: Apart from an accident near the magic size $n=55$, they steadily increase, as expected from simple mesoscopic capillary models [33]. It is worthwhile noting that the size evolution of transition temperatures is correlated, to some extent (once removed the smooth contribution of the latent of evaporation $[34,35]$ ), to the one of dissociation energies (see bottom panel in figure 4), which, in particular, do no longer significantly vary above $n \approx 40[8]$.

Local maxima of the transition temperatures are observed at the "magic sizes" $\left(\mathrm{H}_{2} \mathrm{O}\right)_{21} \mathrm{H}^{+}$and $\left(\mathrm{H}_{2} \mathrm{O}\right)_{55} \mathrm{H}^{+}$. These sizes have high cohesive energies, but their dissociation energies are not necessarily the highest local ones - the dissociation energy of $\left(\mathrm{H}_{2} \mathrm{O}\right)_{20} \mathrm{H}^{+}$is higher than the one of $\left(\mathrm{H}_{2} \mathrm{O}\right)_{21} \mathrm{H}^{+}$, for instance. Nevertheless, a positive correlation between the stability of clusters and their melting temperature is observed in most cases - even though no simple relation can be drawn in the general case [36].

The transition temperatures are plotted in figure 5.a) as a function of experimental dissociation energies [8]. The correlation between both parameters can be split in two parts: Around $n \approx 35$ there is a dramatic break in the curve $T_{m}=f\left(E_{d i s}\right)$ and a change in its slope, which is much higher above $n \approx 35$ than below. $\left(\mathrm{H}_{2} \mathrm{O}\right)_{20} \mathrm{H}^{+}$departs from the general tendency since it has particularly high dissociation energy in regards to its transition temperature. 


\section{Heat capacity of deprotonated water clusters}

The heat capacity of deprotonated water clusters $\left(\mathrm{H}_{2} \mathrm{O}\right)_{\mathrm{n}-1} \mathrm{OH}^{-}$was measured using the same apparatus and the same data processing method than in the case of protonated water clusters. Several examples of the heat capacities of deprotonated clusters that we measured are shown in reference [24]. In figure 4, the size evolution of the transition temperatures of deprotonated water clusters is compared with the results obtained for protonated clusters. For both positively and negatively charged clusters, the size evolution is more regular at large size (roughly, above $n \approx 35$ ) than at small sizes and a singular behavior is observed around $n=55$ in both cases. However, the amplitude of the variations below $n=35$ is less marked for deprotonated species than for protonated ones.

The transitions temperatures of deprotonated water clusters are globally lower and vary with higher amplitude at small sizes than at large sizes. The correlation between dissociation energies and transition temperature is weaker than for protonated clusters. As for protonated clusters, a positive correlation between the binding energies of deprotonated water clusters and their transition temperatures is shown in figure 5.b). Only one component is visible for deprotonated clusters, whereas this correlation was split in two parts for protonated clusters.

\section{Discussion}

\section{Positively charged water clusters}

At least in the size range considered here, the large majority of the positively charged water clusters observed in laboratory experiments are protonated species $\left(\mathrm{H}_{2} \mathrm{O}\right)_{n} \mathrm{H}^{+}$. In the few theoretical studies devoted to the thermodynamics of water clusters $[36,29,30]$, the simulated caloric curves of protonated water clusters show an increase of the heat capacity as in the experiments (although calculations give transition temperatures slightly higher than experiments). Several kinds of phase transitions were proposed to account for this: transition from cage structures to "flower" structures, 
from flower to ring or treelike structures [29], onset of delocalization of the $\mathrm{H}^{+}$ion - that is to say, a gradual transition as the temperature increases from Eigen $\left(\mathrm{H}_{3} \mathrm{O}^{+}\right)$to $\mathrm{Zundel}\left(\mathrm{H}_{2} \mathrm{O} \cdots \mathrm{H}^{+} \cdots \mathrm{OH}_{2}\right)$ form $[30]$.

The very nature of the phase transitions revealed here remains unknown. Electronic effects are not expected to play a major role here, and the transitions are certainly due to structural changes. They may be either a glass transition, solid-solid structural transitions or a genuine melting transition [17]. Let us briefly recall what is known about the structure of protonated water clusters.

Clathrate-like cage structure are suggested to explain the well-known particular stability of $\left(\mathrm{H}_{2} \mathrm{O}\right)_{21} \mathrm{H}^{+}$and $\left(\mathrm{H}_{2} \mathrm{O}\right)_{28} \mathrm{H}^{+}[10,36,29,4,37,38,39,40,41,42,43,44] .\left(\mathrm{H}_{2} \mathrm{O}\right)_{21} \mathrm{H}^{+}$is probably a pentagonal dodecahedron $\left(5^{12}\right)$, with the hydronium ion $\mathrm{H}_{3} \mathrm{O}^{+}$occupying a surface state $[42,43,30,45]$, and $\left(\mathrm{H}_{2} \mathrm{O}\right)_{28} \mathrm{H}^{+}$is thought to be a hexakaidecahedron $\left(5^{12} 6^{4}\right)[4]$.

Single cage are no longer the most stable structures above about 35-molecule clusters, which are expected to have more disordered geometries, or to be made of face sharing multiple clathrate cages with one or several water molecules or ions inside [37,46]. Putative global minima found using empirical potentials show a transition around $n=37$ from (more or less distorted) single cage structures to geometries with a small structured inner core [6]. There is so far no definitive structural explanation of the high abundance of $\left(\mathrm{H}_{2} \mathrm{O}\right)_{55} \mathrm{H}^{+}$. It is not necessarily due to its particularly high stability, but might be due to the instability of $\left(\mathrm{H}_{2} \mathrm{O}\right)_{56} \mathrm{H}^{+}$[8]; the high transition temperature of $\left(\mathrm{H}_{2} \mathrm{O}\right)_{55} \mathrm{H}^{+}$, however, is not expected to depend on the stability of the neighboring sizes, and suggests this cluster to have either a higher stability or an entropic advantage in comparison to its neighbors.

Size-selected IR vibrational spectroscopy contributed to shed light on the structure of water clusters in the last decade. By analyzing the band corresponding to 3-coordinated molecules, which is a typical signature of cage structures, IR vibrational spectra support the hypothesis of protonated water clusters having a clathrate-like cage structures at least in the size range $n=21-27[38,39,40]$, with a variable number of water molecules inside. These structures are generally not favored with a large energy advantage, and entropic effects are also likely to play a role [47]. Although some authors supposed 
cage structures to occur around $\mathrm{n} \approx 15[29,48]$, the most recent IR spectroscopy experiments suggest that the appearance of cage structure rather occurs at $n=21$, since the free $\mathrm{OH}$ peak ascribed to 2coordinated water band, still rather intense at $n=20$, becomes suddenly much weaker at $n=21$ $[10,39,49]$.

Let us examine now what information may be brought by the present work about the structure of water clusters, bearing in mind that it is necessarily a speculative approach to some extent, since no direct information about clusters structure is available from nanocalorimetry experiments.

The most visible feature of the correlation between dissociation energies and transition temperatures displayed in figure 5.a) is its splitting in two components corresponding to small and large sizes, respectively. This feature depending on the dissociation energies, whose behavior simultaneously changes around $n \approx 35$, might induce a biased correlation. It is also well known that many properties of clusters evolve from highly size-dependent at small sizes to more monotonous at larger sizes [50]. However, a correlation clearly appears on the graph and deserves to be addressed.

An attempted explanation can be proposed, which rests on a change of structural properties of the clusters around $n \approx 35$, through the following rough analysis. First, one makes the hypothesis (which is actually not directly supported by our experimental results) that there is a first order phase transition at the transition temperatures defined above. Within this assumption, first-order transition temperatures $T_{\text {trans }}(n)$, entropy changes $\Delta S(n)$, and latent heats $L(n)$ are related by the well-known expression $T_{\text {trans }}(n)=1 / \Delta S(n) \times L(n)$. The latent heats $L(n)$ are positively correlated with the dissociation energies $D(n)$ [51,52], which do not show any abrupt variations around $n=35(8,47)$. Therefore, within the frame of this rough model, the slope of the curve $T_{\text {trans }}=f(D)$ is expected to vary as $1 / \Delta S$, and the break at $n \approx 35$ could be linked to a sudden decrease of the transition entropy $\Delta S$. Hence, such behavior may come from an evolution from ordered to less ordered solid structures whose entropy is closer to the one of the liquid state (or to any less ordered structure), thus reducing the transition entropy. The same rationale addresses the peculiarity of $\left(\mathrm{H}_{2} \mathrm{O}\right)_{20} \mathrm{H}^{+}$, which is then expected to be more disordered than cage-like $\left(\mathrm{H}_{2} \mathrm{O}\right)_{21} \mathrm{H}^{+}$. 


\section{Negatively charged water clusters}

Very few studies were devoted, both experimentally and theoretically, to negatively charged water clusters as compared to positively charged ones. Since their first observation in 1981 [9], two kinds of anionic water clusters are currently produced in laboratory experiments, namely $\left(\mathrm{H}_{2} \mathrm{O}\right)_{\mathrm{n}-1} \mathrm{OH}^{-}[8,53,24]$ and $\left(\mathrm{H}_{2} \mathrm{O}\right)_{\mathrm{n}}^{-}[9,11,23]$. In deprotonated species $\left(\mathrm{H}_{2} \mathrm{O}\right)_{\mathrm{n}-1} \mathrm{OH}^{-}$, the charge is assumed to be carried locally by a $\mathrm{OH}^{-}$sub-unit, whereas in $\left(\mathrm{H}_{2} \mathrm{O}\right)_{n}^{-}{ }^{-}$clusters, the electron is more likely solvated.

In the low size range, deprotonated and protonated water clusters behave quite differently. First of all, the very strong magic number observed at $n=21$ for protonated water clusters does not appear at all for negatively charged clusters, which means that negatively charged and positively charged water clusters have different structures at this size. The data we provide here support the hypothesis that protonated water clusters may have different structures than deprotonated water clusters at low sizes. The less rugged profile of the transition temperature size dependence suggests weaker variations of the cluster structure from size to size. Furthermore, and contrary to what is observed for protonated clusters, the size dependence of the cluster binding energies exhibits only one component, supporting the idea that deprotonated clusters do not undergo a size-dependent transition, as protonated do around $n \approx 35$. In addition, the slope of the correlation observed for deprotonated clusters over the whole size range (from $n=20$ to 70 ) is close to the one observed for large protonated clusters which were suggested to have disordered geometries. Grouping these three facts together leads to the hypothesis that deprotonated clusters may have different and less ordered structures below $n \approx 35$ than protonated species.

For larger sizes, the temperature dependences of transition temperatures is rather smooth, insofar as deduced from the reduced number of measurements, except in the vicinity of $n=55$. As for protonated species, there is a sharp local increase, although with moderate amplitude, of the transition temperatures around 55 molecules. This brings and additional proof, if there were ever a need, of the peculiarity of 55 molecules water clusters, but no reason for this can be deduced from our experiment. 


\section{Conclusion}

The heat capacity of size-selected protonated and deprotonated water clusters was measured, for the first time, in an extended size range, between $\sim 60 \mathrm{~K}$ and $\sim 140 \mathrm{~K}$. Well-defined peaks, signature of first order phase transitions, cannot be observed in water clusters. Nevertheless, for both charge states, the heat capacity of water clusters shows the same feature: A slow increase at low temperatures followed by a sharper increase at a size dependent temperature at which clusters are likely to undergo a phase or structural transition. Such a behavior of water cluster's caloric curves was also qualitatively observed in theoretical works; however, no comprehensive and reliable theoretical caloric curves are available in the size range investigated in the present work, whose analysis must therefore rely on speculative hypothesis.

The main features of the phase transitions observed in protonated water clusters can be summed up as follows (lay stress on $n \approx 35$ not referring here to an exact value but to a size range of a few molecules around $n=35$ ): $i$ ) Below $n \approx 35$, the size evolution of transition temperatures does not follow any marked global trend. The large fluctuations from size to size are signatures of ordered structures.

ii) Around $n \approx 35$, there is a break in the relation between transition temperatures and dissociation energies. This break is possibly related to a reduction of transition entropy. iii) Above $n \approx 35$, the sizeevolution of transition temperatures is much smoother than below and shows a global increase. These experimental features lead us to draw up the following hypothesis: from 21 to around 35 molecules, protonated water clusters have low entropy ordered structures, probably more or less distorted cage structures with one or two molecules inside, whereas water clusters of more than about 35 molecules have generally a more marked amorphous character.

Contrary to protonated water clusters, the heat capacities of deprotonated water clusters do not provide indication of a size-dependent structural transition. In the size range $n \approx 20-35$, our results suggest that deprotonated water clusters are less structured than protonated clusters since $i$ ) there is no magic sizes at $n=21$, ii) the correlation between binding energies and transition temperatures looks 
like the one of unstructured protonated clusters, and iii) the variations of both transition temperatures and dissociation energies are weaker than for protonated clusters.

\section{Acknowledgments}

This work has been partly funded by the french Agence Nationale de la Recherche (ANR) under Grant No. ANR 2011-BS04-028-01.

[1] D.W.Oxtoby, J. Phys : Condens. Matter 4, 7627-7650 (1992).

[2] I.J. Ford, Phys. Rev. E 56, 5615 (1997).

[3] J. Wölk, R. Strey, J. Phys. Chem. B 105, 11683 (2001).

[4] Ralf Ludwig and Andreas Appelhagen, Angew Chem. Int. Ed. 44, 811 (2005).

[5] Ralf Ludwig, Angew. Chem. Int. Ed. 40, 1808 (2001).

[6] Sergey Kazachenko and Ajit J. Thakkar, J. Chem. Phys. 138, 194302 (2013)

[7] H. Haberland und H. Langosch, Z. Phys. D 2, 243-247 (1986)

[8] K. Hansen, P. U. Andersson, and E. Uggerud, J. Chem. Phys. 131, 124303 (2009)

[9] Martin Armbruster, Hellmut Haberland, and Hans-Georg Schindler, Phys. Rev. Lett. 47, 323 (1981)

[10] Kenta Mizuse, Naohiko Mikami, and Asuka Fujii, Angew. Chem. Int. Ed. 49, 10119-10122 (2010)

[11] Lei Ma, Kiran Majer, Fabien Chirot, and Bernd von Issendorff, J. Chem. Phys. 131, 144303 (2009)

[12] G. Torchet, J. Farges, M. F. de Feraudy, and B. Raoult, Ann. Phys. Fr. 14, 245 (1989).

[13] Christoph C. Pradzynski, Richard M. Forck, Thomas Zeuch, Petr Slavíček, Udo Buck, Science 337, 1529 (2012).

[14] Jan K. Kazimirski and Victoria Buch, J. Phys. Chem. A 107, 9762-9775 (2003).

[15] M. Schmidt, R. Kusche, B. von Issendorff, and H. Haberland, Nature (London) 393, 238 (1998). 
[16] G. A. Breaux, R. C. Benirschke, T. Sugai, B. S. Kinnear, and M. F. Jarrold, Phys. Rev. Lett. 91, 215508 (2003).

[17] M. Schmidt and B. von Issendorff, J. Chem. Phys. 136, 164307 (2012).

[18] F. Chirot, P. Feiden, S. Zamith, P. Labastie, and J.-M. L’Hermite, J. Chem. Phys. 129, 164514 (2008).

[19] G. A. Breaux, C. M. Neal, B. Cao, and M. F. Jarrold, Phys. Rev. B 71,073410 (2005).

[20] G. A. Breaux, B. Cao, and M. F. Jarrold, J. Phys. Chem. B 109, 16575 (2005).

[21] C. M. Neal, A. K. Starace, and M. F. Jarrold, Phys. Rev. B 76, 054113 (2007).

[22] G. A. Breaux, R. C. Bernischke, and M. F. Jarrold, J. Chem. Phys. 121, 6502 (2004).

[23] C. Hock, M. Schmidt, R. Kuhnen, C. Bartels, L. Ma, H. Haberland, and B. v. Issendorff, Phys. Rev. Lett. 103, 073401 (2009)

[24] Sébastien Zamith, Pierre Labastie, and Jean-Marc L'Hermite, J. Chem. Phys. 138, 034304 (2013)

[25] Sébastien Zamith, Pierre Feiden, Pierre Labastie, and Jean-Marc L'Hermite, Phys. Rev. Lett. 104, 103401 (2010)

[26] Sébastien Zamith, Pierre Feiden, Pierre Labastie, and Jean-Marc L'Hermite, J. Chem. Phys. 133, 154305 (2010)

[27] E. Uggerud and P. J. Derrick, J. Phys. Chem. 95, 1430 (1991)

[28] M. F. Jarrold and E. C. Honea, J. Phys. Chem. 95, 9181 (1991).

[29] Jer-Lai Kuo, Michael L. Klein, J. Chem. Phys 122, 024516 (2005)

[30] N. Jiten Singh, Mina Park, Seung Kyu Min, Seung Bum Suh, and Kwang S. Kim, Angew. Chem. Int. Ed. 118, 3879 (2006).

[31] D. M. Murphy and T. Koop, Q. J. R. Meteorol. Soc. 131, pp. 1539-1565 (2005)

[32] A. Angell, Science 319, 582 (2008)

[33] P. Labastie and F. Calvo, in Thermodynamics and Solid-Liquid Transition, Nanomaterials and Nanochemistry, edited by C. Bréchignac, C. P. Houdy, and M. Lahmani (Springer, New York, 2007).

[34] G. A. Breaux,D.A. Hillman, C.M. Neal, R.C. Benirschke, and M. Jarrold, J. Am. Chem. Soc. 126, 8628 (2004)

[35] E.G. Noya, J.P.K. Doye, D.J. Wales and A. Aguado, Euro. Phys. J. D. 43, 57-60, (2007)

[36] J. Douady, F. Calvo, and F. Spiegelman, Eur. Phys. J. D 52, 47-50 (2009) and references therein

[37] Sang-Won Lee, Patrick Freivogel, Thomas Schindler, and J. L. Beauchamp, J. Am. Chem. Soc. 120, 11758 (1998).

[38] J.-W. Shin et al., Science 304, 1137 (2004).

[39] M. Miyazaki, A. Fujii, T. Ebata, N. Mikami, Science 304, 1134 (2004).

[40] Kenta Mizuse and Asuka Fujii, J. Phys. Chem. Lett. 2, 2130-2134 (2011).

[41] Timothy S. Zwier, Science 304, 1119 (2004). 
[42] Chih-Che Wu, Chih-Kai Lin, Huan-Cheng Chang, Jyh-Chiang Jiang, Jer-Lai Kuo, Michael L. Klein, J. Chem. Phys. 122, 074315 (2005).

[43] Arshad Khan, Chemical Physics Letters 319, 440-450 (2000).

[44] Bernhard Bandow and Bernd Hartke, J. Phys. Chem. A 110, 5809-5822 (2006).

[45] Srinivasan S. Iyengar, Matt K. Petersen, Tyler J. F. Day, and Christian J. Burnham, Virginia E. Teige, Gregory A. Voth, J. Chem. Phys. 123, 084309 (2005).

[46] Arshad Khan, J. Phys. Chem. A 103, 12604 (1999).

[47] Z. Shi, J. V. Ford, S. Wei, and A. W. Castleman, Jr., J. Chem. Phys. 99, 8009 (1993).

[48] Matthew P. Hodges, David J. Wales, Chem. Phys. Lett. 324, 279 (2000).

[49] Kenta Mizuse, Asuka Fujii, and Naohiko Mikami, J. Chem. Phys. 126, 231101 (2007).

[50] Joshua Jortner, Z. Phys. D - Atoms, Molecules and Clusters 24, 247-275 (1992)

[51] A. K. Starace, C. M. Neal, B. Cao, M. Jarrold, A. Aguado, J. M. López, J. Chem. Phys. 129, 144702 (2008).

[52] S. Zamith, F. Chirot and J.-M. L'Hermite, Europhysics Letters 92, 13004 (2010).

[53] Sébastien Zamith, Grégoire de Tournadre, Pierre Labastie, and Jean-Marc L'Hermite, J. Chem. Phys. 138, 034301 (2013) 


\section{Figure captions}

Figure 1. Barycenter $\bar{n}$ plotted as a function of the number of collisions $\bar{i}$ (bottom scale) or water vapor pressure in the collision cell (top scale). The data are obtained by size selecting $\left(\mathrm{H}_{2} \mathrm{O}\right)_{118} \mathrm{H}^{+}$ clusters at a kinetic energy of $33 \mathrm{eV}$ in the laboratory frame, corresponding to a $\mathrm{CM}$ collision energy $E_{c}=0.32 \mathrm{eV}$. The experiment was repeated at different initial temperatures of the clusters $(\mathrm{T}=47,97$ and $127 \mathrm{~K}$, respectively, from top to bottom). The number of collisions is obtained from the water vapor pressure assuming a geometric cross-section. The vertical line indicates the working pressure in the experiments used to determine the caloric curves of $\left.\mathrm{H}_{2} \mathrm{O}\right)_{118} \mathrm{H}^{+}$. The derivatives $\partial \bar{n} / \partial \bar{i}\left(T_{t h 1}\right)$ and $\partial \bar{n} / \partial \bar{i}\left(T_{t h 2}\right)$ are used to evaluate the corrective term in relation (1) (see text).

Figure 2. Raw data used for determining the heat capacity of mass selected water clusters, here exemplified for $\left(\mathrm{H}_{2} \mathrm{O}\right)_{55} \mathrm{H}^{+}$: The barycenter of the mass distribution $(\bar{n}$, see text $)$ is plotted as a function of cluster's initial temperature at two CM collision energies, namely $E_{c}=0.64 \mathrm{eV}$ (squares) and $E_{c}=0.73 \mathrm{eV}$ (circles) here. Slightly smoothed interpolation of experimental data (continuous lines), were used for calculating the heat capacity shown in the inset using relation (1). The water vapor pressure in the collision cell is here $4 \times 10^{-4}$ mbar, each cluster undergoes on average $\bar{i} \approx 2$ collisions. Inset: Heat capacity $C(T)$ and transition temperature extracted from the two curves above. In the hatched area, the values of $C(T)$ are unreliable owing to the evaporation of clusters before they enter the collision cell. Dotted line : Heat capacity of bulk ice (taken from [31]).

Figure 3. Size-evolution of the heat capacity plotted as a function of temperature of protonated water clusters $\left(\mathrm{H}_{2} \mathrm{O}\right)_{\mathrm{n}} \mathrm{H}^{+}$. 
Figure 4. Top: Transition temperatures as a function of disseciation energies size for protonated (full squares) and deprotonated (open circles) water clusters. Also represented are results previously released by Schmidt et al [17] for protonated clusters and Hock et al [23] for $\left(\mathrm{H}_{2} \mathrm{O}\right)_{\mathrm{n}}{ }^{-}$clusters. Bottom: dissociation energies (taken from reference [8]) of protonated (full squares) and deprotonated (open circles) water clusters.

Figure 5. Transition temperatures plotted as a function of dissociation energies (taken from reference [8]) for $a$ ) protonated and $b$ ) deprotonated water clusters. The slopes (dashed lines) are drawn only as visual guide. The numbers inside squares and circles indicate the size of the clusters. In figure a), squares and circles distinguish small $(n \leq 30)$ and large $(n \geq 35)$ clusters, respectively. 


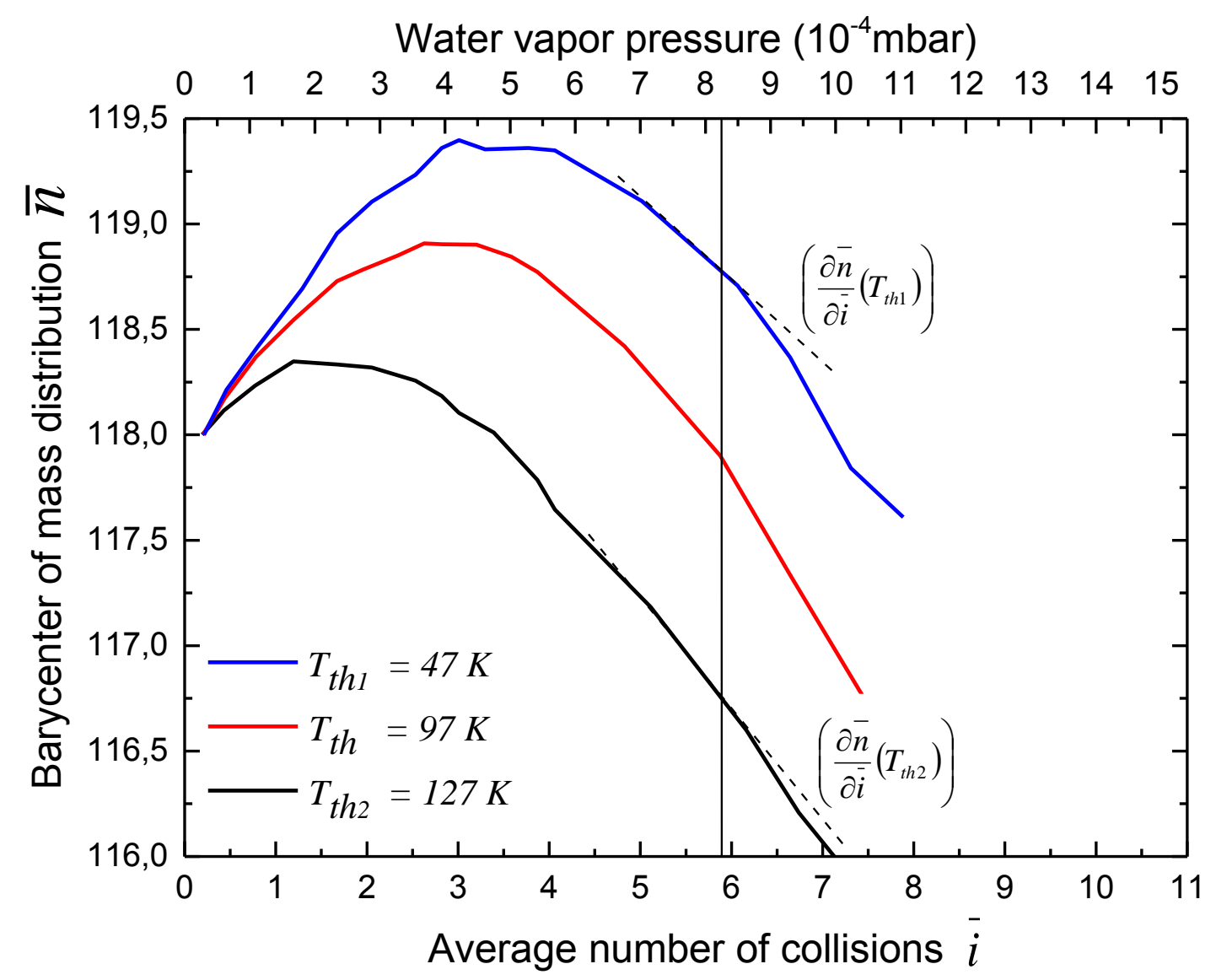

Figure 1 


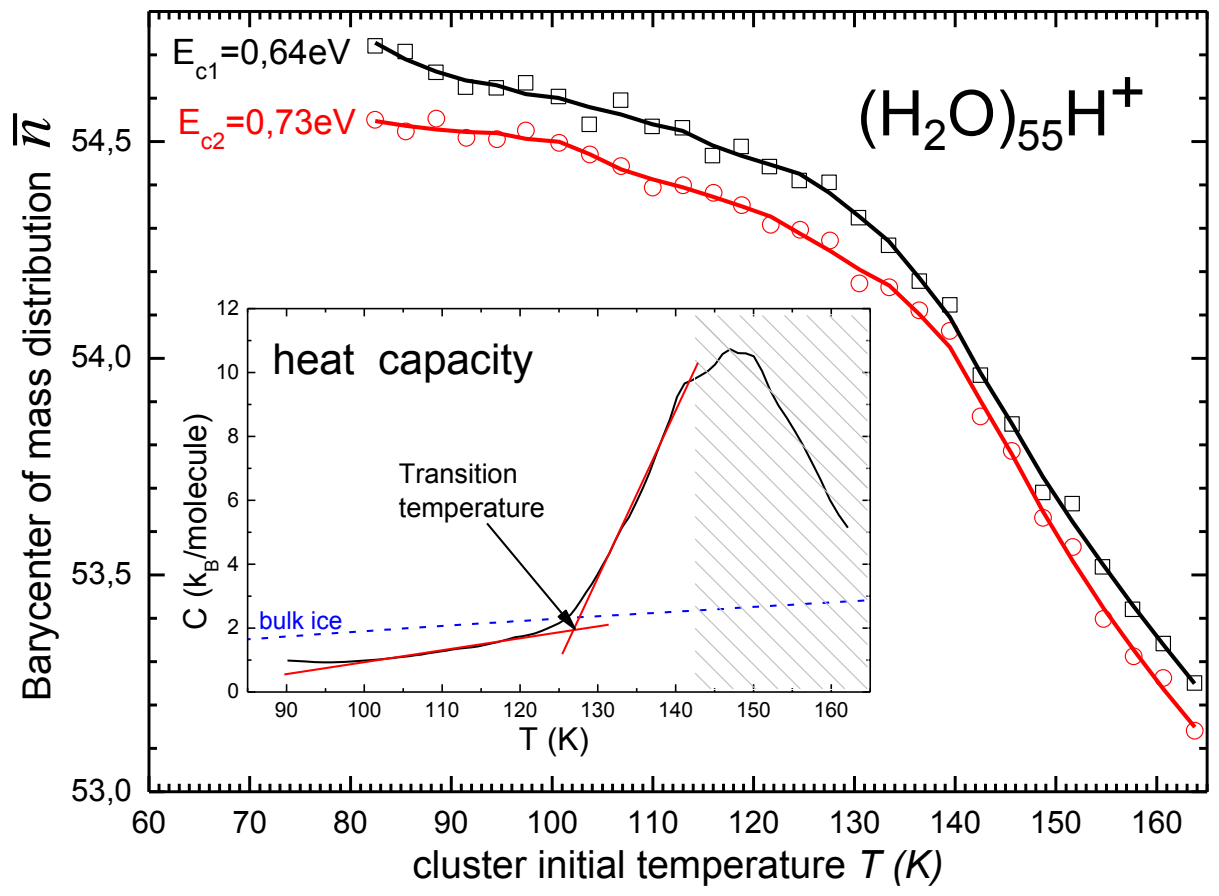

Figure 2 


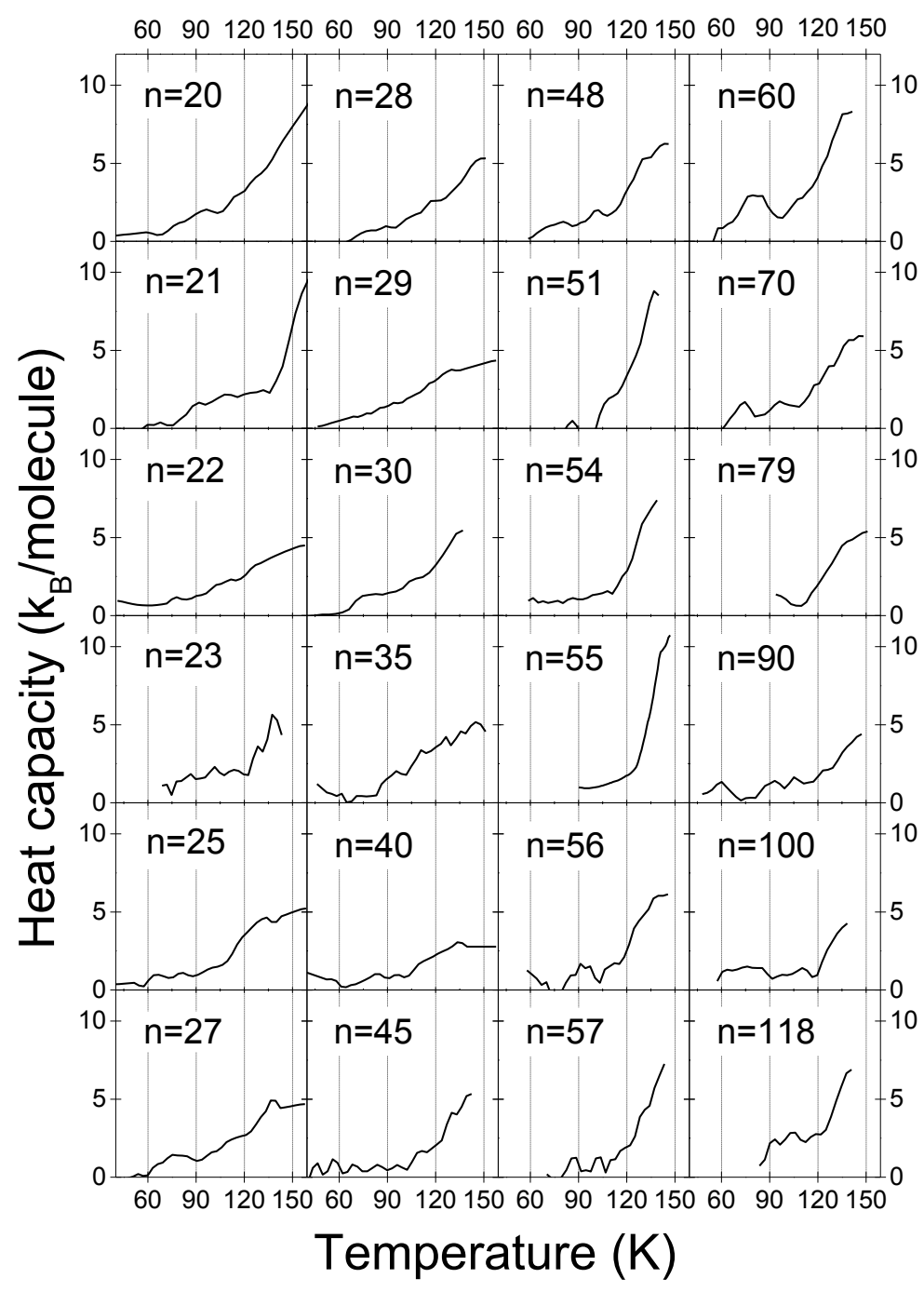

Figure 3 


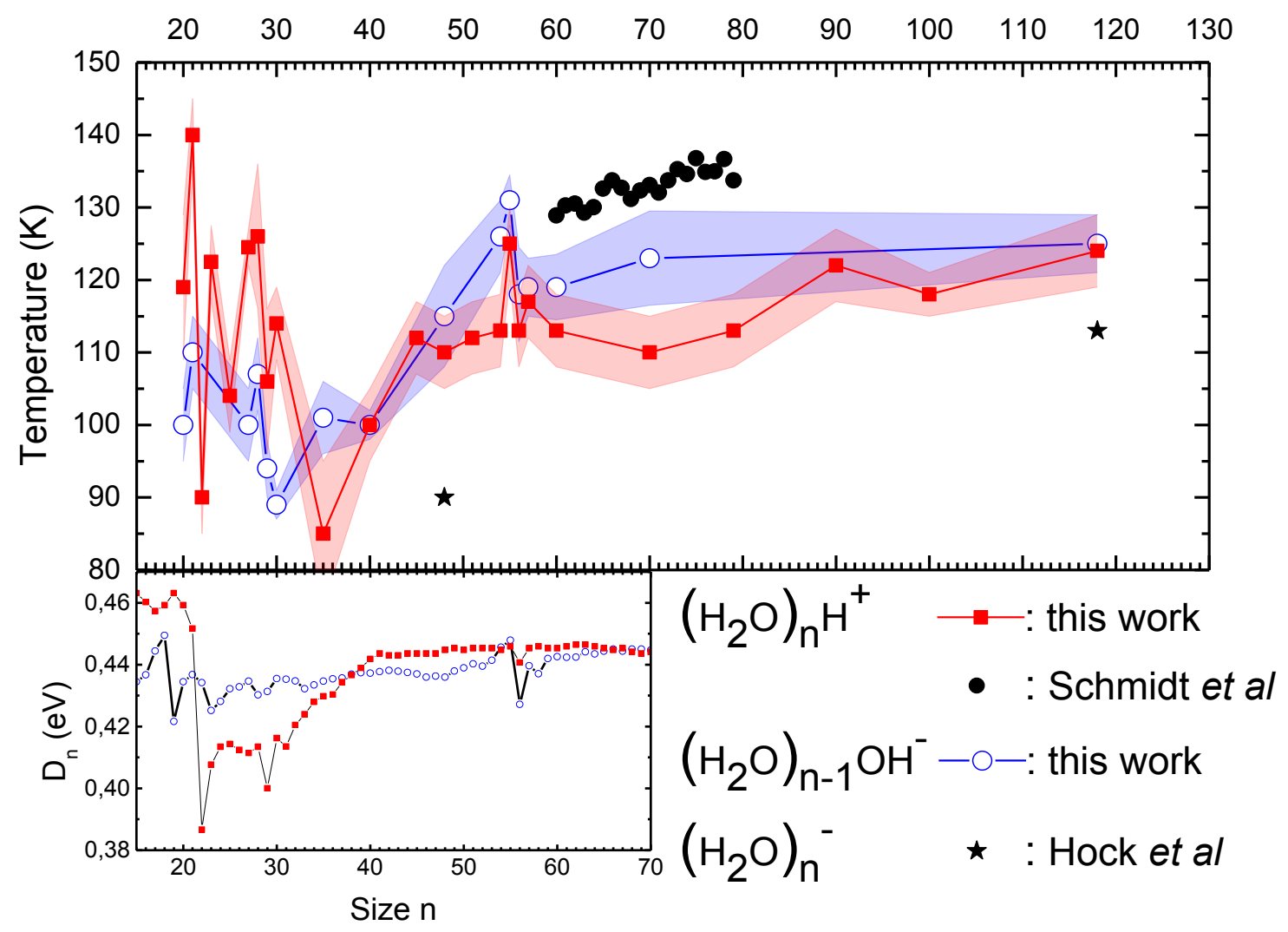

Figure 4 
a)
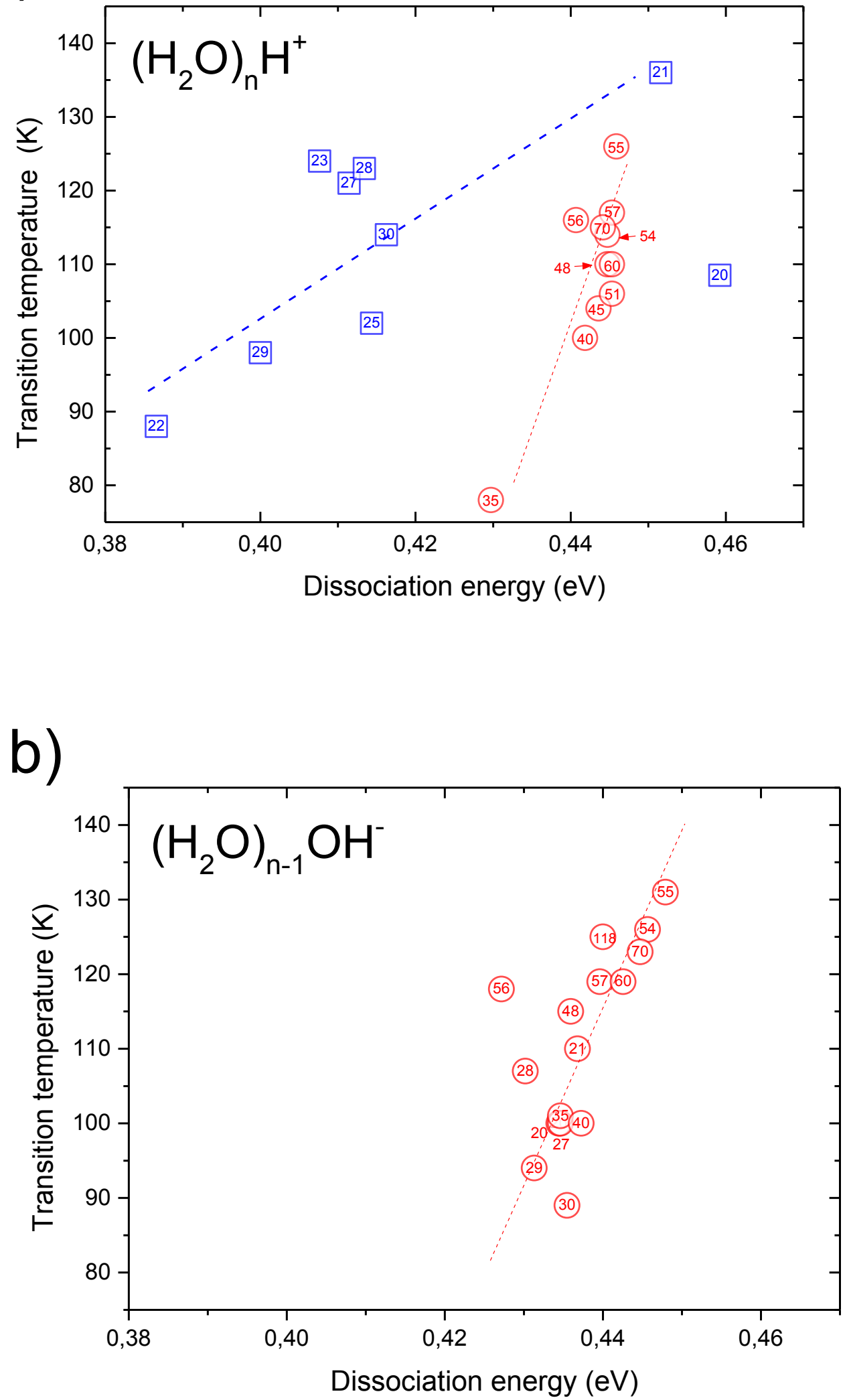

Figure 5 